\title{
Intraosseous Meningioma of Sphenoid Bone
}

\author{
Shaymaa Ahmed Sadek ${ }^{1}$, Sohaila Fatima ${ }^{1 *}$, Nazima Haider ${ }^{1}$ and Balkur Krishnamoorthi Adiga ${ }^{2}$ \\ ${ }^{1}$ Deptt. of Laboratory Medicine, Aseer Central Hospital, Abha , KSA \\ ${ }^{2}$ Deptt. of Pathology, King Khalid University, Abha,KSA
}

\section{ABSTRACT}

Primary intraosseous meningiomas are rare tumors and a subtype of primary extradural meningiomas . We present a 35 years old female with left temporal prominence and proptosis of left eye who was diagnosed as intraosseous meningioma.

Keywords: Intraosseous Meningioma , Extradural, Sphenoid Bone

\section{Introduction}

Meningiomas account for approximately $20 \%$ to $30 \%$ of primary intracranial tumors. They arise from arachnoidal cap cells which are generally situated proximal to the meninges and dura [1] . Primary intraosseous meningiomas (PIM) are a subtype of primary extradural meningiomas (PEM) which constitute less than $2 \%$ of all meningiomas and PIM represent approximately two thirds of all PEM [2].

\section{Case Report}

A 35-year-old female presented with left temporal prominence and proptosis of 8 months duration. Computerised tomographic (CT) scan of brain and orbits with/without contrast revealed expansile sclerotic bony lesion of lesser wing of left sphenoid bone associated with proptosis of left eye [Figure 1]. Multiplanar multisequential magnetic resonance imaging (MRI) pre and post Gadolinium(Gd) administration showed extra axial dural based enhancing mass in left middle cranial fossa pole with enhancement of greater wing of left sphenoid bone, lateral and superior orbital walls . It measured $0.9 \times 2.1 \times 2.9$ $\mathrm{cm}$ in the anteroposterior, transverse and craniocaudal dimension . Left frontal craniotomy was done and tumor tissue was excised Gross examination showed a piece of bone measuring $5 \times 2.5 \times 1.7 \mathrm{~cm}$ with adherent membrane measuring 3x $0.3 \mathrm{~cm}$. Histopathology revealed markedly thickened bony trabeculae with almost total replacement of marrow spaces by proliferating meningothelial cells with indistinct cytoplasmic borders and eosinophilic cytoplasm, regular oval nuclei with occasional intranuclear inclusions [Figure 2,3]. No atypia, mitosis or necrosis was identified. The tumor cells were positive for vimentin, epithelial membrane antigen (EMA) and progesterone receptors (PR) [Figure 4]. A diagnosis of an intraosseous meningioma (meningiotheliomatous WHO Grade I) was made .The patient is on followup and is doing well.

\section{Discussion}

Meningiomas are clinicopathologically diverse group of neoplasms with morphologic, immunohistochemical and ultrastructural features resembling normal meningothelial cells [3]. PEM or ectopic meningioma are a rare locationspecific type of meningioma that arise outside the dural covering of the brain and spinal cord. They are extracranial tumours predominantly located in the head and neck most commonly in the sinonasal tract, temporal bone, ear and in the scalp. IOM is a term used to describe a subset of PEMs that arise in bone [4] .According to Lang et al PEM are classified as purely extracalvarial (type I), purely calvarial (type II), or calvarial with extracalvarial extension (type III) . According to the site of location of the tumor type II and type III lesions are further subdivided into convexity (C) or skull base (B) forms [5]. IOM must be differentiated from bone invasion of a thin, enplaque or carpet like dural based meningioma [1].

The vast majority of PIM arise in cranial bones, although a few cases in which the tumor originated in the mandible have been reported [6]. The convexity ( periorbital region and frontoparietal region) and the skull base are the two primary locations for PIM [2]. They occur with the same frequency in both sexes or with a slight female predominance. These tumors have a bimodal incidence with one peak in the second decade and a second peak during the fifth through seventh decades of life $[2,5]$. Clinical presentation depends on location and varies from incidental detection to neurological deficit, seizures, vomiting, dizziness, hearing loss, tinnitus, headache, vague sensations in the head, cranial nerve deficits, proptosis, deformity, nasal obstruction or epistaxis [2].Our patient presented with headache and proptosis. 


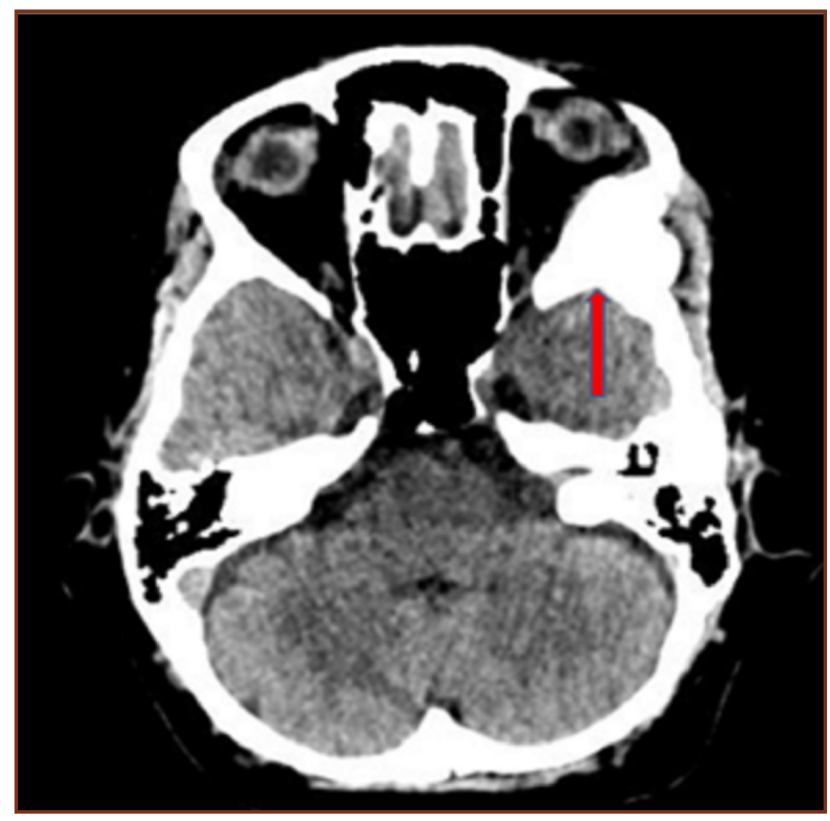

Fig. 1: CT scan showing expansile sclerotic bony lesion (red arrow) of lesser wing of left sphenoid bone associated with proptosis of left eye.

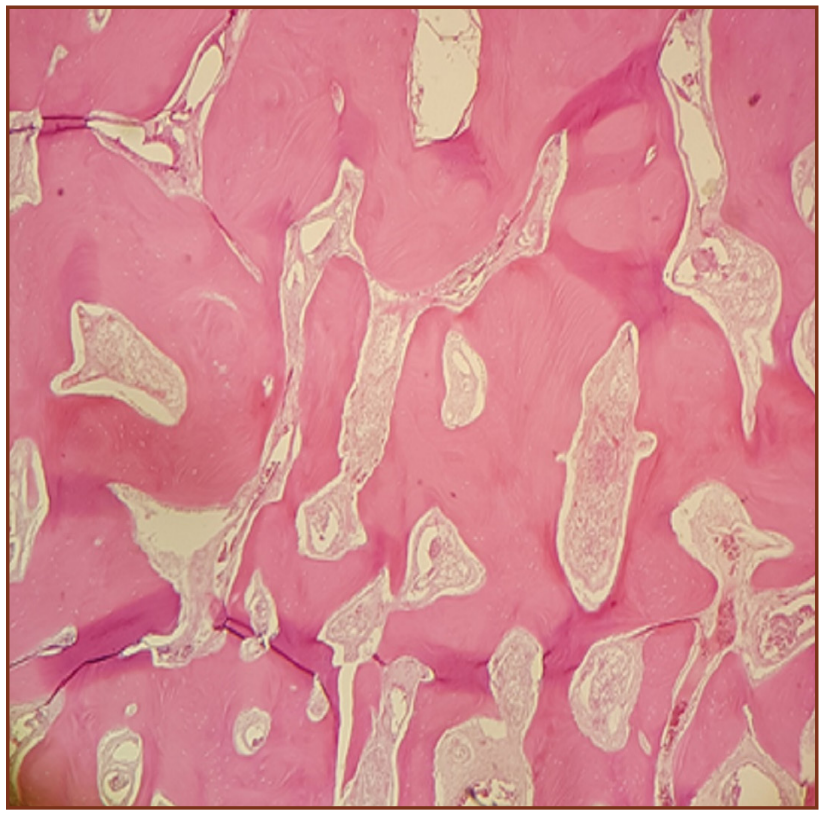

Fig. 2: A. Section showing thickened bony trabeculae with almost total replacement of marrow spaces by proliferating meningothelial cells with indistinct cytoplasmic borders and eosinophilic cytoplasm. (Hematoxylin and Eosin,10X).

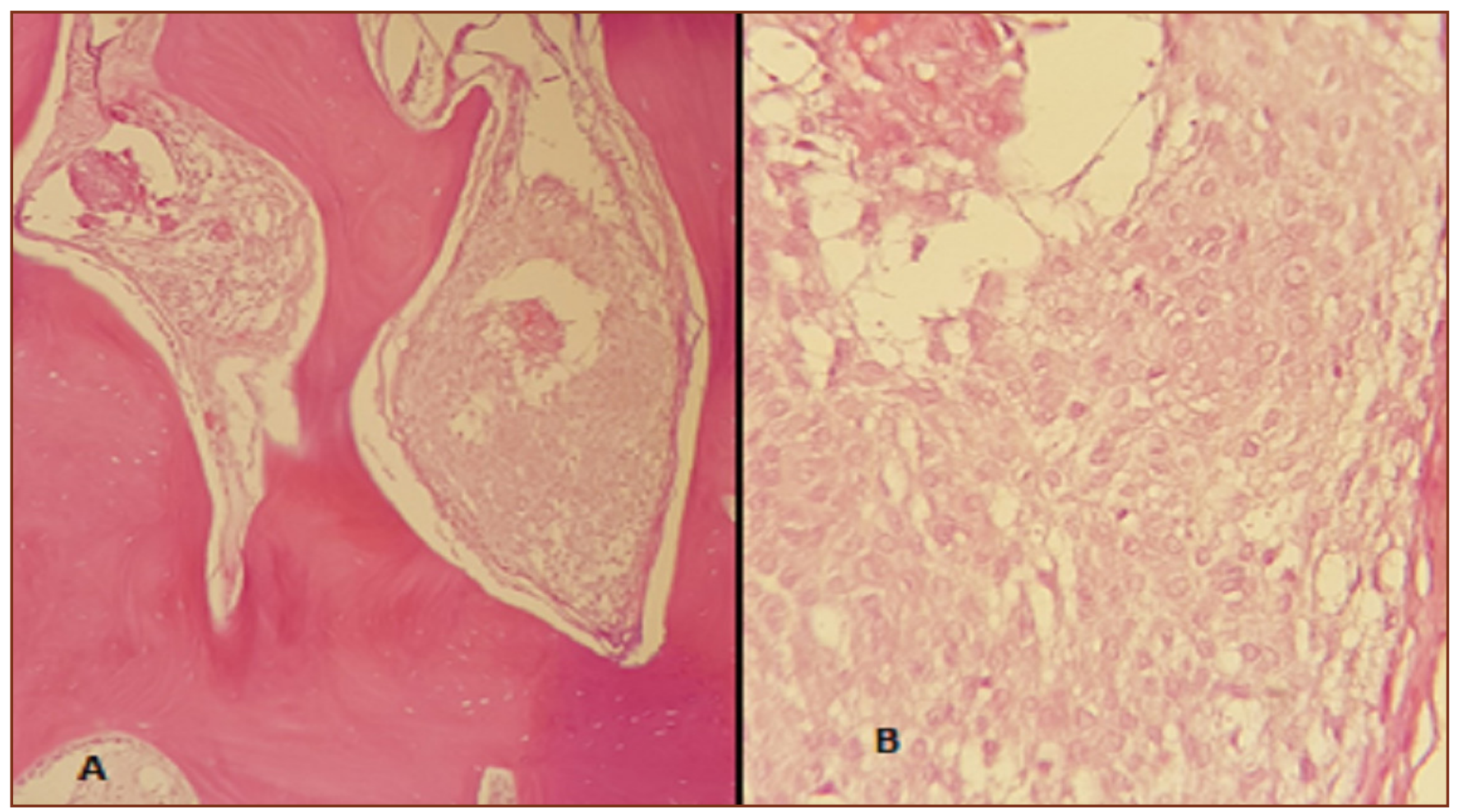

Fig. 3 : A. Section showing bony trabeculae with replacement of marrow spaces by proliferating meningothelial cells B. Meningothelial cells with indistinct cytoplasmic borders and eosinophilic cytoplasm, regular oval nuclei with occasional intranuclear inclusions. (Hematoxylin and Eosin , A- 20X B-40X). 


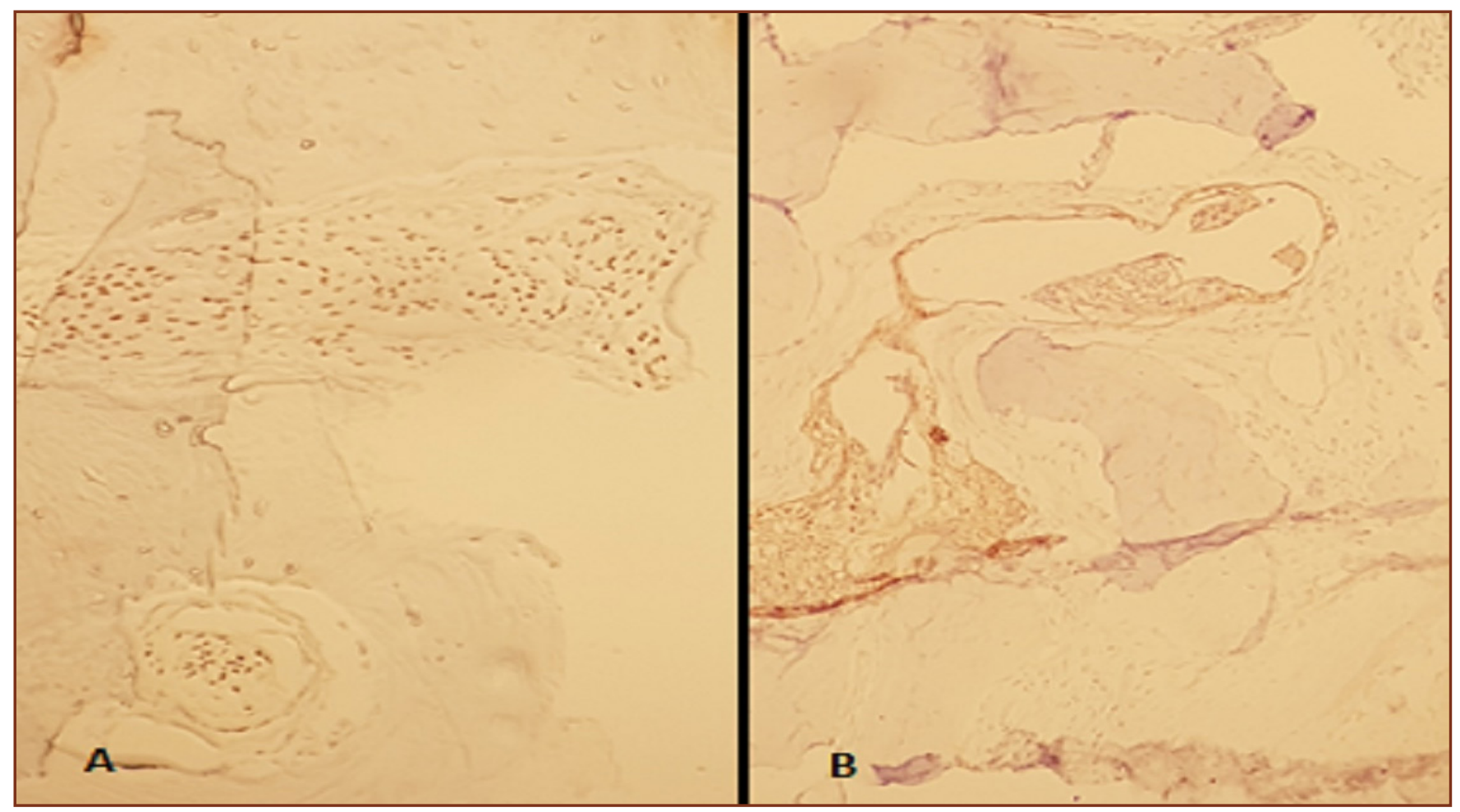

Fig. 4: Immunohistochemical study showing A.PR positivity in tumor cells within marrow spaces B. EMA positivity in tumor cells within marrow spaces ( A-PR x 20X, EMA x 20X).

Crawford and colleagues published a report that reviewed the radiographic findings of the 34 published cases of PIM. Radiographic evidence of hyperostosis was noted in 20 cases $(59 \%)$, whereas $11(32 \%)$ showed osteolytic changes in the surrounding bone.Two cases $(6 \%)$ revealed a mixed picture of both osteolysis and hyperostosis [7].CT with bone windows shows a focally thickened, hyperdense lesion expanding the calvaria. The tumor is usually hyperdense on a nonenhanced CT scan and enhances densely after contrast administration. The expansion of bone and a "ground glass" appearance of this type of PIM may appear radiographically similar to fibrous dysplasia [8]. MRI findings are hypointense on T1-weighted images and hyperintense on T2-weighted images . Prominent homogeneous enhancement after Gd administration is typical. These tumors do not usually exhibit the "dural tail" often found with intradural meningiomas, but $\mathrm{Gd}$ enhancement of the underlying dura may be noted. This dural enhancement could be secondary to dural irritation or tumor invasion [9]. MR imaging is necessary for the evaluation of the soft tissue component and extradural extension [10].

Microscopic pathology often reveals eosinophilic tumor cells with indistinct borders grouped in clusters and whorls. The nuclei are typically oval and regular and nuclear pseudoinclusions may be observed [3]. The bone may appear normal, with replacement of the marrow by fat, fibrosis, and tumor cells.In a study the majority of PIM $(62.5 \%)$ were of the meningothelial type; transitional ( $25 \%$ ), fibroblastic ( $8.3 \%$ ), psammomatous ( $2.1 \%$ ) and malignant ( $2.1 \%$ ) [11]. Large majority of meningiomas exhibit immunolabeling for EMA,PR [3].

Meninges are mesenchymal in origin; meningiomas may develop from multipotential mesenchymal cell precursors, thereby accounting for the occurrence of meningiomas in uncommon locations[12]. Other explanations such as head trauma, abnormal cranial molding or embryogenesis, and arachnoid cells accompanying blood vessels or cranial nerves as they traverse the skull could be other explanations for the origin of an PIM depending on the clinical and radiographic presentation [2].

The treatment of choice is resection of the mass which is potentially curative. Tumors that cannot be completely resected may require adjuvant therapy which may include radiation therapy, chemotherapy, or bisphosphonate therapy. The majority of these tumors are slow-growing and long-term follow-up is required to rule out recurrent tumors. Recurrence of atypical or malignant PIM has been reported to occur up to 24 months after resection, whereas histologically benign tumors have been reported to recur up to 120 months after surgery [5]. Broeckx et al developed 
a simple technique to enable single-step resection of a skull lesion and cranial reconstruction thereby reducing surgical time and costs and ensuring a good cosmetic result [13].

In conclusion PIM is a subtype of PEM .It is a rare indolent tumor and surgical resection is the treatment of choice.

\section{References}

1. Ellison D, Love S, Chimelli LMC , Harding BN, Lowe JS , Vinters HV, Brandner S, Yong WH.Neuropathology: A Reference Text of CNS Pathology, 3ed: 2012,Elsevier. 9780723435150

2. Elder JB, Atkinson R, Zee CS, Chen TC. Primary intraosseous meningioma. Neurosurg Focus 2007;23:E13. [Crossref]

3. Rosai and Ackerman's Surgical Pathology. 11th ed. vol 2. Mosby St. Louis, Missouri; 2017.

4. Politi M, Romeike BF, Papanagiotou P, Nabhan A, Struffert $\mathrm{T}$, Feiden $\mathrm{W}$, et al. Intraosseous hemangioma of the skull with dural tail sign: radiologic features with pathologic correlation. AJNR Am J Neuroradiol 2005; 26: 2049-52.

5. Lang FF, Macdonald OK, Fuller GN, DeMonte F. Primary extradural meningiomas: a report on nine cases and review of literature from the era of computerized tomography scanning. J Neurosurg 2000;93:940-50.

6. Lell M, Tudor C, Aigner T, Kessler P: Primary intraosseous meningioma of the mandible: CT and MR imaging features. AJNR Am J Neuroradiol 28:129-131, 2007.
7. Crawford TS, Kleinschmidt-DeMasters BK, Lillehei KO: Primary intraosseous meningioma. Case report. J Neurosurg 83:912-915,1995.

8. Changhong L, Naiyin C, Yuehuan G, Lianzhong Z: Primary intraosseous meningiomas of the skull. Clin Radiol 52:546550, 1997.

9. Arana E, Diaz C, Latorre FF, Menor F, Revert A, Beltrán A, et al:Primary intraosseous meningiomas. Acta Radiol 7:937-942,1996.

10. Ilica AT, Mossa-Basha M, Zan E, Vikani A, Pillai JJ, Gujar S, Aygun N, Izbudak I. Cranial intraosseous meningioma; spectrum of neuroimaging findings with respect to histopathological grades in 65 patients. Clin Imaging. 2014;38:599-604.View ArticlePubMedGoogle Scholar

11. Yamazaki T, Tsukada A, Uemura K, Satou H, Tsuboi K, Nose T:Intraosseous meningioma of the posterior fossaCase report.Neurol Med Chir (Tokyo) 41:149-153, 2001.

12. Shuangshoti S, Netsky MG, Fitz-Hugh GS. Parapharyngeal meningioma with special reference to cell of origin. Ann Otol Rhinol Laryngol 1971;80:464-473.

13. Broeckx CE ,Maal TJJ, Vreeken RD, Bos RRM, Laan M.Single-Step Resection of an Intraosseous Meningioma and Cranial Reconstruction: Technical Note.World Neurosurgery 2017;108 ，225 - 9. DOI: https://doi. org/10.1016/j.wneu.2017.08.177

*Corresponding author:

Dr. Sohaila Fatima, Deptt. of Pathology, King Khalid University, Abha,KSA.

Phone: +91 966-502184094

Email: sohailafatima@gmail.com

Financial or other Competing Interests: None. 